\title{
Dysphagia: a growing concern? a personal view
}

\begin{abstract}
The population across the world is ageing. The fastest growing group is that of the $>85$ years. Many will be frail, have multiple long term conditions and dysphagia. Inevitably this will result in malnutrition, hospital admissions and death. Action needs to be taken to prevent this happening by concerted action from Public Health. Failure to do this will result in a strain on health resources.
\end{abstract}

"Death is caused by swallowing small amounts of saliva over a long period of time". George Carlin (1937-2008)

Keywords: hospital, health resources, world health organization, constipation, strength
Volume 9 Issue 4 - 2018

\section{David G Smithard}

Queen Elizabeth Hospital and University of Greenwich, London

Correspondence: David G Smithard, Queen Elizabeth Hospital and University of Greenwich, Avery Hill,Woolwich, Greenwich, London, Email david.smithard@hotmail.nhs

Received: April 0I, 2018 | Published: August 02, 2018

\section{Introduction}

The world's population is growing and it is predicted that by 2050 , $30 \%$ of the population will be $>65$ years of age. ${ }^{1}$ People $>85$ years are the fastest growing cohort in Europe and will soon be $30 \%$ of all people aged $>65$ years of age. Many of the very old ( $>85$ years) older people are not healthy in old age, as defined by the World Health Organization; ${ }^{2}$ many will have one or more long-term condition such as diabetes, heart disease, lung disease, osteoarthritis and osteoporosis.

As the numbers of very old people increase, the numbers that are frail and disabled will also increase. Frailty is a complex syndrome $^{3,4}$ associated with sarcopenia, under-nutrition, slowness, fatigue, low energy expenditure and frequently disability. Frail older people have multiple co-morbidities (long term conditions), a limited physiological reserve, ${ }^{5,6}$ and increased mortality. ${ }^{7}$ Any insult (constipation, environmental change, infection, trauma, medication change) can result in decompensation, due to the poor physiological reserve, ${ }^{5,6}$ which will result in a reduction in physical and cognitive functioning and may result in the inability to swallow.

Sarcopenia manifests as a loss of muscle bulk and strength, with a change in muscle fibresresulting in a loss of contractility. These changes are seen in skeletal muscle, particularly appendicular muscles. Sarcopenia usually associated with poor nutrition. From an older person's perspective there are two reasons why nutritional intake may be compromised. There may be limited access to food (poor mobility, no shop, no bus) and any food obtained is of poor nutritional quality. Secondly eating and drinking may be compromised (presbyphagia, neurological disease, rheumatoid arthritis,) due to a poor swallow. ${ }^{8,9}$ Fifty percent of those $>80$ years of age will have sarcopenia, resulting in weakness of the supra-hyoid muscles and the pharyngeal stripping muscles and hence a slow swallow and dysphagia.

Eating and drinking go hand in had with swallowing. Food needs to be transferred to the mouth. If there weakness, deformity (Rheumatoid arthritis; Stroke) are present then it is difficult to cut food up and then transfer it to the mouth (unable to move the arms, difficulty holding a cup or cutlery). Once the food is in the mouth, it will need to be chewed and pass through the mouth, to the pharynx and thence to the oesophagus. ${ }^{10}$

\section{Swallowing}

The International Classification of Functioning, Disability and Health (ICF 2001) classify swallowing as "functions of clearing the food and drink through the oral cavity, pharynx and oesophagus into the stomach at an appropriate rate and speed". The oral phase of swallowing needs strong oral facial and tongue muscles to prepare the bolus. ${ }^{11}$ Therefore if teeth are painful or missing, chewing is difficult. Dentures are often not worn due to discomfort. Dental plates may become infected with candida resulting in recurrent oral thrush and pain when swallowing if the pharynx is involved. The food will need to be manipulated by the tongue, its function may be reduced due to neurological disease (stroke) malignancy or infection. ${ }^{12,13}$

The pharynx is a joint pathway for air and food. During the swallow the larynx is protected, so that food and drinks do not go down the wrong way, which may result in aspiration. For a safe swallow the larynx is pulled up and forward, the tongue moves back towards the pharynx and the pharynx has a peristaltic wave to push food through the larynx to the oesophagus. During this time the upper oesophageal sphincter needs to relax to let food and drink pass through. The elevation and anterior movement of the larynx is dependent on the function of extrinsic muscles (the supra-hyoid group). The muscles are weak in frail older people and do not lift the larynx or pull it forward enough, nor do they pull the UES open as much. The consequence of this is that the airway is at risk before, during and after the swallow. Before because the poor elevation of the larynx may result in it failing to close, during due to poor tongue retraction and poor pharyngeal peristalsis and after due to increased pharyngeal residue due to poor UES opening. ${ }^{14}$

Dysphagia is not infrequent in those residing in the community. Various studies have noted a frequency of up to $30 \%$ of older people living at home,,$^{15} 28 \%$ of ole people undergoing instrumentation (FEES;Videofluoroscopy) are shown to aspirate. ${ }^{16,17}$ With increasing dependency and illness the prevalence of dysphagia increases (Figure $1)$.

Older people may not present with dysphagia, yet may have subtle changes within their swallow mechanism, which they have not noticed and have unconsciously compensated for; ${ }^{5}$ if they have accepted it is as a natural consequence of getting older. ${ }^{18}$ Fatigue is commonly 
present in frail older people, food intake is reduced because ittakes too long to long eat, and fatigue may worsen their swallow as muscles tire with use.

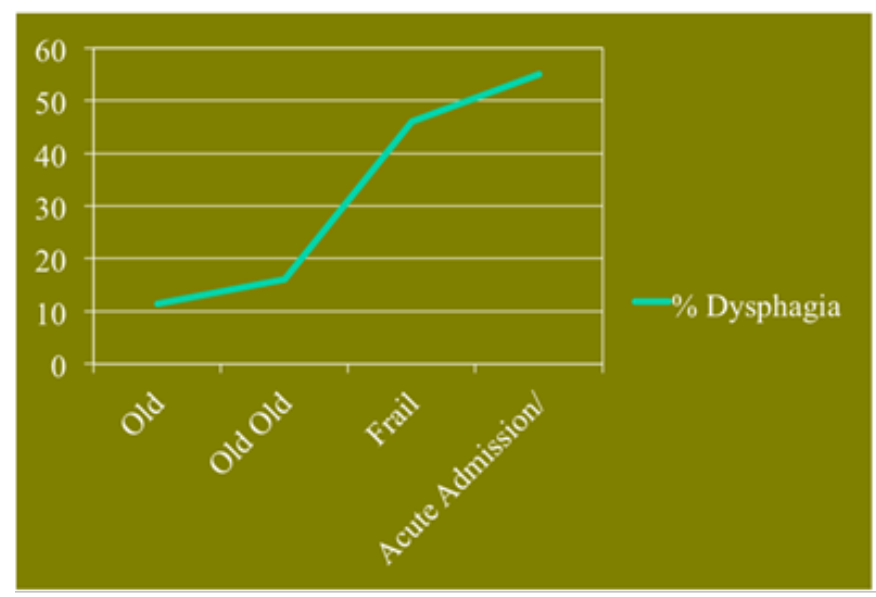

Figure I Increasing dependency is associated with increased prevalence of dysphagia.

\section{Identification of dysphagia}

With the ageing population, the incidence and prevalence of dysphagia and its complications are going to become a major problem. Dysphagia is being increasingly recognized as a geriatric syndrome. ${ }^{8,9}$ Safe swallowing has not been recognised as a priority for frail older people care. Acute stroke patients will have their swallow early during their admission as any delay in full assessment is associated with an increased risk of death. ${ }^{19}$ Dysphagia and potential aspiration is a great risk in older people. Especially those those are very frail and old.

The literature is reflecting the new found awareness that difficulty swallowing is common in many old people. Able et al (unpublished) showed that many 80-90 year olds will fail a 3ox water test. The prevalence of dysphagia post intubation on ITU ${ }^{20}$ and after surgery ${ }^{21}$ is greater than previously recognized. Similar to other disease episodes, the presence of dysphagia is associated increases in length of stay and mortality

\section{Pro-active screening}

As people age, admission to hospital with pneumonia is increasingly associated with aspiration, frequently associated with poor mouth care rather than the aspiration of food. Despite this older people are not routinely assessed to see if they can swallow. Stroke patients, in many countries, are assessed at the time for admission as it is recognised that aspiration pneumonia is a significant problem. Dysphagia is an independent marker of mortality, morbidity and length of hospital stay and admission to a care home on discharge from hospital. Every time a frail older person is admitted to hospital with delirium secondary to any infection their functional status declines and never returns to preadmission levels resulting in increasing dependency.

\section{Hospital admission}

Many older people are immune-compromised because of medication (steroids/Methotrexate), malignancy, poor diet, presence of diabetes increasing their risk of infection and a consequent poor prognosis. Pneumonia is a common problem, resulting in many hospital admissions and deaths amongst older people each year. ${ }^{22}$ This is recognised in the UK such that vaccinations against influenza are offered yearly and against pneumococcus five yearly to those most at risk of infection (old people, lung conditions, immune compromised).

With increasing age and increasing frailty there is an increased prevalence of aspiration pneumonia rather than community acquired pneumonia, such that $80 \%$ of those $>90$ will have aspiration pneumonia; frequently associated with poor mouth care rather than the aspiration of food (ref) and mortality may be as high as $34 \%, 23$ readmission to hospital is also increased..$^{24}$ Despite this older people are not routinely assessed to see if they can swallow. Stroke patients, in many countries, are assessed at the time for admission as it is recognised that aspiration pneumonia is a significant problem. ${ }^{2}$ Dysphagia is an independent marker of mortality, morbidity and length of hospital stay and admission to a care home on discharge from hospital. Every time a frail older person is admitted to hospital with delirium secondary to any infection their functional status declines and never returns to preadmission levels resulting in increasing dependency.

\section{Complications}

Dysphagia has psychosocial (isolation, fear of eating) complications as much as medical (chest infection, malnutrition, pressure ulcers, falls) complications. From a public health perspective the major issues are the potentially increasing older population and the inadequate way in which there is a proactive approach taken.

\section{Public health}

The role of public health is to keep people from illness. From a frailty and dysphagia point of view, a proactive approach needs to be taken. Old people, particularly in health care settings (hospital, care home), need their swallow assessed at the time of admission to that facility. Failure will result in malnutrition, chest infection26, dependency and institutionalization and the economic strain put on health care and social services is not inconsequential. An admission to hospital is expensive, because of increased care needs and increased hospital stay. In the USA, Patel et al, found that total inpatient costs were a mean $\$ 6243$ higher among those with a dysphagia diagnosis (\$19244 vs 13001, $\mathrm{P}<0.001) 26$.

\section{Conclusion}

If awareness is raised, the correct questions asked, dysphagia can be recognized and managed early. The question arises as to which bedside screening assessments should be used. At present there are many, different assessments that are used, some validated, some not. This approach will only support staff and residents in care homes; a full population approach is needed within the community to raise awareness with the population and health care staff, to prevent dysphagia occurring.

Given that sarcopenia plays a pivotal role exercises of the extrinsic muscles could be encouraged. A second approach may be to encourage a total body approach resulting in the toning of many muscles. (This may be cheaper and easy, but is as yet untested. A more targeted approach utilizes chin tuck exercises such as Chin Tuck against resistance. Laryngeal exercises and head raises are other techniques that have been suggested, but only used after dysphagia has been identified. At this moment in time there are more questions than answers. We need to find the answers to support the health of older people. 


\section{Acknowledgements}

None.

\section{Conflict of interest}

The author declares no conflict of interest.

\section{References}

1. http://ea.uk.org/unpd/wpp/Graphs/DemographicProfiles.

2. World Report on Ageing and Health 2015. USA:WHO; 2015.

3. Fried LP, Tangem CM, Walsten J, et al. Frailty in older adults: evidence for a phenotype. J Gerontology A Biol Sci Med Sci. 2001;56(3):M146-157

4. Phu S, Boersma D, Duque G. Exercise and sarcopenia. J Clin Densitom. 2015;18(4):488-492

5. Omari TI, Kritas S, Cock C, et al. Swallowing dysfunction in healthy older people using pharyngeal pressure-flow analysis. Neurogastroenterol Motil. 2014;26(1):59-68.

6. Nicosia MA, Hind JA, Roecker EB, et al. Age effects on the temporal evolution of isometric and swallowing pressure. J Gerontol A Biol Sci Med Sci. 2000;55(11):M634-640.

7. Stanaway FF, Gnjidic D, Blyth FM, et al. Hoe fast does the Grim Reaper walk? Receiver operating characteristics curve analysis in healthy men aged 70 and over. BMJ. 2011;343:d7679.

8. BajensLW, Clavé P, Cras P, et al. European society for swallowing disorders- European Union geriatric medicine society white paper: oro-pharyngeal dysphagia as a generic syndrome. Clin Interv Aging. 2016;11:1403-1428.

9. Smithard DG. Dysphagia: a geriatric giant. Med \&Clin Reviews. 2016;5:10.21767/2471-299X.

10. Dejaeger M, Liesenborghs, Dejaeger E. Presbyphagia. Seminars in Dysphagia. 2015.

11. Otsuka R, Matsui C, Tange C, et al. What is the best adjustment of appendicular an mass for predicting mortality or disability among Japanese community dwellers? BMC Geriatrics. 2018;18(1):8.

12. Humbert IA, Robbins JA. Dysphagia in the elderly. Arch Phys Med Rehabil. 2008;19(4):853-866.

13. Nogueira D, Reis E. Swallowing disorders in nursing home residents: how can the problem be explained? Clin Interv Aging. 2013;8:221-227

14. Chichero JAY, Halley PJ. Variations in the normal swallow. In J Cichero, et al. editors. Dysphagia: Foundation, Theory and Practice. Chichester UK: John Wiley and Sons; 2002.
15. Kertscher B, Speyer R, Fong E, et al. Prevalence of oro-pharyngeal dysphagia in the Netherlands: a telephone survey. Dysphagia. 2015;30(2):114-120.

16. Almirall J, Rofes L, Serra-Prat M, et al. Oropharyngeal dysphagia is a risk factor for community-acquired pneumonia in the elderly. Eur Respir $J$. 2013;41(4):923-928.

17. Butler SG, Stuart A, Leng X, et al. Factors influencing aspiration during swallowing in healthy older adults. Laryngoscope. 2010;120(11):21472152 .

18. Chen PH, Golub JS, Hapner ER, et al. Prevalence of perceived dysphagia and quality of life impairment in a geriatric population. Dysphagia. 2009;24(1):1-6.

19. Bray B, Smith CJ, Cloud GC, et al. The association between delays in screening for and assessing dysphagia after acute stroke, and the risk of stroke-associated pneumonia. J NeurolNeurosurg Psychiatry. 2017;88(1):25-30.

20. Brodsky Mb, Huang M, Shanholtz C, et al. Recovery from dysphagia symptoms after oral endotracheal intubation in acute respiratory distress syndrome survivors. A 5-year longitudinal study. Annals Am Thoracic Soc. 2017;14(3):376-383.

21. Love AL, Cornwell PL, Whitehouse SL. Oro-pharyngeal dysphagia in an elderly post-operative hip fracture population: a prospective cohort study. Age and Ageing. 2013;42(6):782-785.

22. Marik PE, Kaplan D. Aspiration and dysphagia in the elderly. Chest. 2003;124(1):328-336.

23. Melgaard d. The prevalence of oro-pharyngeal dysphagia in acute geriatric patients. Conference proceedings. DRS 2016, Milan; 2016.

24. Melgaard D, Baandrup U, Bøgsted M, et al. Rehospitalisation and mortality after hospitalisation for oro-pharyngeal dysphagia and community acquired pneumonia: a 1-year follow-up study. Geriatric Medicine. 2017.

25. National Clinical Guidelines for Stroke. 4th ed. RCP London: Royal College of physicians; 2012. p. 1-175.

26. Paillaud E, Herbaud $S$, Caillet $P$, et al. Relations between undernutrition and nosocomial infections in elderly patients. Age Ageing. 2005;34(6):619625 .

27. Patel DA, Krishnaswami S, Steger E, et al. Economic and survival burden of dysphagia among inpatients in the United States. Dis esophagus. 2018;31(1):1-7. 